\title{
Correlación entre personalidad patológica y conducta delictiva en población penitenciaria*
}

\author{
Correlation between Pathological Personality \\ and Criminal Behavior in Prison Population
}

\author{
Karen Viviana Salinas Atuesta** \\ ORCID: 0000-0002-5448-8553

\section{Yenny Salamanca Camargo***} \\ ORCID: 0000-0002-0928-8907 \\ Universidad Pedagógica y Tecnológica de \\ Colombia, Tunja, Colombia
}

Recibido: 22 enero de 2018 Revisado: 8 de septiembre de 2018 Aceptado: 4 de junio 2019

\section{Resumen}

Estudio no experimental, de tipo descriptivo-correlacional, cuyo objetivo fue determinar la relación existente entre los patrones de personalidad patológica y la conducta delictiva, en 127 participantes hombres, con rango de edad entre 20 y 59 años y pertenecientes a un centro penitenciario de Colombia; a partir de la aplicación del Inventario Clínico Multiaxial de Millon-III (MCMI-III), en su adaptación española y la revisión de la tipología de la conducta delictiva, establecida en el Código Penal Colombiano. Los resultados a partir del coeficiente de correlación ETA indicaron una correlación significativa, entre los patrones patológicos compulsivo (.67), autodestructivo (.66), y evitativo (.64), con la conducta delictiva. Adicionalmente, se determinó que el $44.6 \%$ puntuó en más de dos escalas; los patrones clínicos narcisista y compulsivo, la patología grave de la personalidad paranoide, el síndrome clínico bipolar y el síndrome clínico grave de trastorno delirante se identificaron como los más prevalentes y se discutió su relación de acuerdo al tipo de delito.

Palabras clave: trastornos de la personalidad; Psicología Criminal; Prisiones.

Artículo de investigación. Citar como: Salinas, K. \& Salamanca, C. Y. (2020). Correlación entre personalidad patológica y conducta delictiva en población penitenciaria. Diversitas: Perspectivas en Psicología, 16(1), 131-142. Dol: https://doi.org/10.15332/22563067.5545

Karen Viviana Salinas Atuesta. Grupo de Investigación Clínica y Salud, Universidad Pedagógica y Tecnológica de Colombia, Tunja. Correo electrónico: karensalinas01@hotmail.com

*.* Autor de correspondencia: Yenny Salamanca Camargo. Docente Escuela de Psicología y Coordinadora Grupo de Investigación Clínica y Salud, Universidad Pedagógica y Tecnológica de Colombia, Tunja. Dirección postal: Calle 24 n. ${ }^{\circ}$ 5-63, Antiguo Hospital San Rafael, Tunja (Boyacá, Colombia) cP. 150002. Correo electrónico: yenny.salamanca@uptc.edu.co 


\section{Abstract}

The aim of this non-experimental, descriptive-correlational study was to determine the relation between patterns of pathological personality and criminal behavior among 127 male participants aged between 20 and 59 and incarcerated in a Colombian correctional facility by applying the Millon Clinical Multiaxial Inventory-III (MCMI-III) in its Spanish version and reviwing the typology of the criminal conduct stipulated in the Colombian Criminal Code. The results from the ETA correlation coefficient indicated a significant correlation between the compulsive (.67), self-destructive (.66), and avoidant (.64) pathological patterns with criminal behavior. Additionally, it was determined that $44.6 \%$ fell under more than two scales; narcissistic and compulsive clinical patterns, severe pathology of paranoid personality, bipolar clinical syndrome, and severe clinical syndrome of delusional disorder were identified as the most prevalent and their relation was discussed according to the type of crime.

Keywords: personality disorders; criminal psychology; prisons.

\section{Introducción}

De acuerdo con los planteamientos de Rodríguez, López y Pueyo (2002), en los años 60 y hasta los 80 no existían modelos psicológicos que explicaran la variable de trastornos de personalidad en relación con la conducta delictiva. Los estudios iniciales se delimitaban a los factores sociales y ambientales para explicar el desencadenamiento y mantenimiento de los actos criminales. Sólo es hasta cuando Kononchuck (1981, citado por Marco, Benítez, \& Morera, 2006) realiza las primeras aproximaciones al desarrollo de modelos explicativos para estas variables, señalando que las personas que presentan rasgos patológicos de personalidad muestran una incapacidad para encauzar adecuadamente los conflictos.

Rodríguez-Arrebola (2005) refiere que el estudio de los trastornos de la personalidad probablemente se ha convertido en uno de los principales temas de investigación dentro del área clínica. Por su parte, De Barbenza (2003), Soria (2006) y Esbec y Echeburúa, (2010), han encontrado una relación directa entre los trastornos de personalidad y la conducta criminal, dado que moldean y afectan el comportamiento del delincuente, convirtiéndose en factores determinantes de la motivación del delito, el tipo de víctima y el modus operandi (González-Guerrero, 2007).
Al analizar los antecedentes empíricos relacionados con trastornos de personalidad en población penitenciaria, se encuentran los estudios de Sáez y Fernández (2000) quienes encontraron una prevalencia de trastornos de personalidad del $90.08 \%$; identificando además, que el trastorno antisocial presenta una co-ocurrencia del $58.01 \%$ con otros trastornos. Así mismo, Vidal, Acosta y Caridad (2004) identificaron que el $36 \%$ de los reclusos cumplen los criterios para trastornos de personalidad; Vicens (2006) en su estudio con población psiquiátrica penitenciaria, encontró que cerca el $40 \%$ cuentan con un trastorno de personalidad. En este mismo sentido, López-Barrachina, Lafuente y García-Latas (2007) identificaron un índice de prevalencia de trastornos de la personalidad del $69.3 \%$.

En cuanto a la presencia de al menos un trastorno de personalidad, Echauri, Fernández-Montalvo, Martínez, y Azcárate (2011) encontraron la presencia en un $79.3 \%$ de la muestra. Vicens-Pons (2009) por su parte, en el $80 \%$ y Fernández-Montalvo y Echeburúa (2008) en $86.8 \%$ : Por su parte, Calvo, Soler, Día y Ventura (2008) encontraron que el diagnóstico principal y secundario más prevalente es el trastorno de personalidad con un $22 \%$ y $11.9 \%$ respectivamente y Casares-López et al. (2010) identificaron un porcentaje elevado de individuos que presentan un patrón de personalidad antisocial $(84.7 \%)$ y dependiente (42.7\%). 
Específicamente en Latinoamérica, Sáez y Silva (2000), encontraron una prevalencia de trastornos de personalidad del $\mathbf{9 0 . 0 8 \%}$ en población penitenciaria de Chile. En ese mismo sentido, Echeverry, Escobar Martínez, Garzón y Gómez (2002) reportaron que entre el $39 \%$ y el $50 \%$ de los condenados por homicidio en México presentan un trastorno de personalidad antisocial y en Colombia, Echeverry et al. (2002), en su estudio sobre la prevalencia de enfermedad mental, homicidio y su relación con trastorno de personalidad antisocial, encontró una prevalencia de rasgos de personalidad antisocial del $50 \%$ en los condenados por homicidio y tentativa de homicidio.

Debido a la escasa evidencia empírica identificada en Colombia, surge el interés por realizar estudios en población penitenciaria que permitan determinar la prevalencia de trastornos de personalidad y su relación con la conducta criminal, para lo cual, se plantea como objetivo de este estudio determinar la relación existente entre patrones de personalidad patológica y la conducta delictiva en reclusos de un centro penitenciario de Colombia.

\section{Método}

\section{Diseño}

La presente fue una investigación de tipo cuantitativo con diseño no experimental, lo cual indica que no existió manipulación de variables, sino observación de los fenómenos en su ambiente natural. El diseño fue de tipo descriptivo-correlacional, ya que se buscó describir el estado actual de las variables, especificando propiedades, características y rasgos importantes del fenómeno de estudio, al igual que la relación entre las variables y de corte trasversal, debido a que se recolectó la información de las variables en un momento especifico (Sampieri, Collado, \& Baptista, 2010).

\section{Participantes}

La muestra estuvo conformada por 127 reclusos, seleccionados a partir de un muestreo probabilístico, estratificado y una distribución proporcional, teniendo en cuenta la asignación de números aleatorios y la utilización de la fórmula:

$$
n=\left(Z^{\wedge} 2\right)^{*} N^{*} p^{*}(1-p) /\left(\left(e^{\wedge} 2\right)^{*}(N-1)+\left(Z^{\wedge} 2\right)^{*} p^{*}(1-p)\right)
$$

En donde, $Z$ representa el nivel de confianza, el cual se estableció del 95\%, E significa el margen de error aceptado, siendo este del $5 \%$ y $\mathrm{N}$ establece el tamaño de la población. Además, se identificó una probabilidad (p) del $90 \%$, a partir de una prueba piloto, la cual consistió en la aplicación del instrumento de recolección de datos a 10 participantes, en esta se encuentra que 9 de cada 10 puntúan presencia de algún trastorno de personalidad. Esta probabilidad, además se sustenta con las prevalencias del $90 \%$ identificadas en las investigaciones de Sáez y Fernández (2000) y Fernández-Montalvo y Echeburúa (2008).

Teniendo en cuenta que según los índices modificadores de sinceridad del inventario de personalidad, se identificó un comportamiento de respuesta problemático en seis (6) participantes, se hizo necesario descartar dichos protocolos, quedando conformada la muestra por 121 participantes de los cuales 86 participantes se clasificaron dentro del subgrupo de delitos violentos y 35 en el subgrupo delitos no violentos, con un rango de edad entre 20 y 59 años, escolaridad entre primaria y bachillerato en mayor proporción y procedentes de diferentes departamentos de Colombia.

\section{Instrumentos}

\section{Inventario Clínico Multiaxial de Millón-III, (MСMI-III)}

Test diseñado por Millon, Davis, \& Millon (2009), en su adaptación española (2. ${ }^{a}$ edición), ofrece una medida de la personalidad patológica en adultos entre los 18 a 65 años, consta de 175 ítems de tipo verdadero/falso, distribuido en 11 patrones clínicos de la personalidad (esquizoide, evitativo, depresivo, dependiente, histriónico, narcisista, antisocial, agresivo, compulsivo, negativista), 3 rasgos patológicos (esquizotípico, limite, paranoide), 7 síndromes de gravedad moderada (trastorno de ansiedad, trastorno somatomorfo, trastorno bipolar, trastorno distímico, dependencia de alcohol, dependencia de sustancias, trastorno de estrés postraumático), 3 síndromes de gravedad severa (trastorno de pensa- 
miento, depresión mayor, trastorno delirante) y 4 escalas de control (sinceridad, deseabilidad social, devaluación y validez). Cuenta con una fiabilidad test-retest entre 0.84 y 0.96 y una consistencia interna superior a 0.80 . Al evaluar la consistencia interna de cada escala, se evidencia que la validación del cuestionario tiene una fiabilidad adecuada, superando en más de 21 escalas el valor de coeficientes alfa 0.75 . Las escalas que evalúan sinceridad muestran una consistencia interna en deseabilidad social del 0.80 y en devaluación del 0.92 .

\section{Conducta delictiva}

El código penal colombiano divide los delitos en 18 categorías generales, las cuales según el planteamiento de Vázquez (2012), y para efectos de esta investigación, se agrupan en delitos violentos y delitos no violentos. En el primer subgrupo, se hace referencia a los delitos violentos, en este se ubican los delitos contra el régimen constitucional y legal, contra la familia, contra la libertad individual y otras garantías, contra la libertad, integridad y formación sexuales, contra la seguridad pública, contra la vida y la integridad personal, contra personas y contra los bienes protegidos por el derecho internacional humanitario. El segundo subgrupo se denomina delitos no violentos, entre estos se encuentran los delitos contra la salud pública, contra el orden económico social, contra el patrimonio económico, contra la administración pública, contra la eficaz y recta impartición de justicia, contra los derechos de autor y contra la fe pública (Corte Constitucional Colombiana, 2000).

\section{Procedimiento}

En primer lugar, se seleccionó el centro de reclusión en el cual se llevaría a cabo el estudio y se solicitó la respectiva autorización para realizar la investigación. Posteriormente, se contactó a los participantes seleccionados a partir de un muestreo probabilístico, estratificado y una distribución proporcional, teniendo en cuenta la asignación de números aleatorios, a los cuales se les explicó la naturaleza del estudio, y, se estableció el consentimiento informado para participar en la investigación, finalmente se procedió a la aplicación de los cuestionarios por grupos de 20 en una única sesión de 30 minutos.

El análisis estadístico, se realizó mediante el programa estadístico sPss 23.0 y a partir de un análisis descriptivo en primera instancia, se dio un análisis nominal por intervalo (ETA), y a partir de este, se determinó el grado se asociación existente entre una variable cuantitativa de intervalo y una variable categórica nominal (Martin, Cabero, \& Santana, 2008; Pedroza \& Dicovskyi, 2006; Sampieri et al., 2010). De igual forma, se planteó un análisis correlacional entre escalas a través del coeficiente de correlación de Spearman o de Pearson, dependiendo de la normalidad de los datos.

\section{Consideraciones Éticas}

De acuerdo con la ley 1090 de 2006 sobre el ejercicio profesional del psicólogo y la resolución 008430 de 1993, mediante la cual se regula la investigación en salud en Colombia, este estudio se basó en los derechos y principios éticos de respeto, intimidad y dignidad, asegurando la confidencialidad y el anonimato de los participantes (título $\mathrm{I}$, artículo $2^{\circ}$, título VII, capítulo VII, artículos 49 - 51, 55 y 56); de igual forma se rigió por estipulado por el Departamento Administrativo de Ciencia, Tecnología e Innovación (Colciencias), en la ley 1286 de 2009 (acuerdo N. ${ }^{\circ} 0001$, título ॥, articulo 11). Previo a la vinculación de los participantes, se estableció contacto con las directivas y la profesional psicóloga del centro penitenciario, a fin de dar a conocer el objetivo y los alcances de la investigación y, a su vez, contar con su revisión y aval.

\section{Resultados}

En relación con los datos obtenidos en la prueba de trastornos de la personalidad, en cuanto a los índices modificadores de sinceridad los 121 participantes obtuvieron una puntuación de cero, lo cual indica que el protocolo fue válido, y se puede realizar un óptimo estudio de este. En cuanto al índice de deseabilidad social, el $69 \%$ de la muestra presentó puntuaciones mayores a 75 , según esto, los individuos mostraron una tendencia a presentarse de forma favorable o personalmente atractiva, razón por la 
cual se tuvo cuidado al momento de analizar estos datos, ya que los individuos pudieron haber ocultado algún aspecto de sus dificultades psicológicas o interpersonales. Finalmente, los datos obtenidos en el índice de devaluación indicaron que el 19\% de la población presentó una puntuación mayor a 75, por lo cual, se puede concluir que en promedio los individuos no tendían a despreciarse o devaluarse.
Respecto a los datos obtenidos en las escalas, se presenta la descripción de las puntuaciones obtenidas en los patrones clínicos de personalidad, en la Tabla 1, en donde, se evidencia que las prevalencias más altas se ubican en los patrones narcisistas, seguido del compulsivo.

Tabla 1.

Patrones clínicos de personalidad según conducta delictiva

\begin{tabular}{|c|c|c|c|c|c|c|c|}
\hline \multirow{3}{*}{$\begin{array}{l}\text { Patrones clínicos } \\
\text { de personalidad }\end{array}$} & \multirow{3}{*}{ Puntuación } & \multicolumn{6}{|c|}{ Tipo de delito } \\
\hline & & \multicolumn{2}{|c|}{ Violento } & \multicolumn{2}{|c|}{ No violento } & \multicolumn{2}{|c|}{ Total } \\
\hline & & $\mathbf{F}$ & $\%$ & $F$ & $\%$ & $\mathbf{F}$ & $\%$ \\
\hline \multirow{3}{*}{ Esquizoide } & $<75$ & 85 & 98.8 & 32 & 91.4 & 117 & 96.7 \\
\hline & $75-84$ & 1 & 1.2 & 3 & 8.6 & 4 & 3.3 \\
\hline & $>85$ & 0 & 0.0 & 0 & 0.0 & 0 & 0.0 \\
\hline \multirow{3}{*}{ Evitativa } & $<75$ & 85 & 98.8 & 35 & 100 & 120 & 99.2 \\
\hline & $75-84$ & 1 & 1.2 & 0 & 0.0 & 1 & 0.8 \\
\hline & $>85$ & 0 & 0.0 & 0 & 0.0 & 0 & 0.0 \\
\hline \multirow{3}{*}{ Depresiva } & $<75$ & 85 & 98.8 & 34 & 97.1 & 119 & 98.3 \\
\hline & $75-84$ & 1 & 1.2 & 1 & 2.9 & 2 & 1.7 \\
\hline & $>85$ & 0 & 0.0 & 0 & 0.0 & 0 & 0.0 \\
\hline \multirow{3}{*}{ Dependiente } & $<75$ & 86 & 100.0 & 35 & 100 & 121 & 100 \\
\hline & $75-84$ & 0 & 0.0 & 0 & 0.0 & 0 & 0.0 \\
\hline & $>85$ & 0 & 0.0 & 0 & 0.0 & 0 & 0.0 \\
\hline \multirow{3}{*}{ Histriónica } & $<75$ & 82 & 95.3 & 32 & 91.4 & 114 & 94.2 \\
\hline & $75-84$ & 2 & 2.3 & 2 & 5.7 & 4 & 3.3 \\
\hline & $>85$ & 2 & 2.3 & 1 & 2.9 & 3 & 2.5 \\
\hline \multirow{3}{*}{ Narcisista } & $<75$ & 36 & 41.9 & 13 & 37.1 & 49 & 40.5 \\
\hline & $75-84$ & 20 & 23.3 & 6 & 17.1 & 26 & 21.5 \\
\hline & $>85$ & 30 & 34.9 & 16 & 45.7 & 46 & 38.0 \\
\hline \multirow{3}{*}{ Antisocial } & $<75$ & 81 & 94.2 & 33 & 94.3 & 114 & 94.2 \\
\hline & $75-84$ & 5 & 5.8 & 2 & 5.7 & 7 & 5.8 \\
\hline & $>85$ & 0 & 0.0 & 0 & 0.0 & 0 & 0.0 \\
\hline \multirow{3}{*}{ Agresiva (sádica) } & $<75$ & 86 & 100.0 & 35 & 100 & 121 & 100.0 \\
\hline & $75-84$ & 0 & 0.0 & 0 & 0.0 & 0 & 0.0 \\
\hline & $>85$ & 0 & 0.0 & 0 & 0.0 & 0 & 0.0 \\
\hline \multirow{3}{*}{ Compulsiva } & $<75$ & 50 & 58.1 & 30 & 85.7 & 80 & 66.1 \\
\hline & $75-84$ & 23 & 26.7 & 4 & 11.4 & 27 & 22.3 \\
\hline & $>85$ & 13 & 15.1 & 1 & 2.9 & 14 & 11.6 \\
\hline \multirow{3}{*}{$\begin{array}{l}\text { Negativista (pasivo- } \\
\text { agresivo) }\end{array}$} & $<75$ & 86 & 100.0 & 34 & 97.1 & 120 & 99.2 \\
\hline & $75-84$ & 0 & 0.0 & 0 & 0.0 & 0 & 0.0 \\
\hline & $>85$ & 0 & 0.0 & 1 & 2.9 & 1 & .8 \\
\hline \multirow{3}{*}{ Autodestructiva } & $<75$ & 86 & 100.0 & 35 & 100 & 121 & 100.0 \\
\hline & $75-84$ & 0 & 0.0 & 0 & 0.0 & 0 & 0.0 \\
\hline & $>85$ & 0 & 0.0 & 0 & 0.0 & 0 & 0.0 \\
\hline
\end{tabular}

Nota. $\mathrm{F}<75=$ Ausencia; $\mathrm{F} 75-84=$ Presencia de rasgo; $\mathrm{F}>85=$ Prominencia del síndrome; $\%=$ porcentaje

Fuente: elaboración propia. 
En cuanto a las patologías graves de personalidad, como se puede analizar en la Tabla 2, la mayor prevalencia se ubica en paranoide $(63 \%)$.

Tabla 2.

Patología grave de la personalidad según conducta delictiva

\begin{tabular}{|c|c|c|c|c|c|c|c|}
\hline \multirow{3}{*}{$\begin{array}{l}\text { Patología grave de } \\
\text { la personalidad }\end{array}$} & \multirow{3}{*}{ Puntuación } & \multicolumn{6}{|c|}{ Tipo de delito } \\
\hline & & \multicolumn{2}{|c|}{ Violento } & \multicolumn{2}{|c|}{ No violento } & \multicolumn{2}{|c|}{ Total } \\
\hline & & $\mathbf{F}$ & $\%$ & $\mathrm{~F}$ & $\%$ & $\mathbf{F}$ & $\%$ \\
\hline & $<75$ & 25 & 29.1 & 19 & 54.2 & 44 & 36.4 \\
\hline \multirow[t]{3}{*}{ Paranoide } & $75-84$ & 52 & 60.4 & 12 & 34.3 & 64 & 52.9 \\
\hline & $>85$ & 9 & 10.5 & 4 & 11.5 & 13 & 10.1 \\
\hline & $<75$ & 85 & 98.8 & 34 & 97.1 & 119 & 98.3 \\
\hline \multirow[t]{3}{*}{ Esquizotípica } & $75-84$ & 1 & 1.2 & 1 & 2.9 & 2 & 1.7 \\
\hline & $>85$ & 0 & 0.0 & 0 & 0.0 & 0 & 0.0 \\
\hline & $<75$ & 85 & 98.8 & 35 & 100.0 & 120 & 99.2 \\
\hline \multirow[t]{2}{*}{ Límite } & $75-84$ & 1 & 1.2 & 0 & 0.0 & 1 & 0.8 \\
\hline & $>85$ & 0 & 0.0 & 0 & 0.0 & 0 & 0.0 \\
\hline
\end{tabular}

Nota. $\mathrm{Fr}<75$ = Ausencia; Fr $75-84=$ Presencia de rasgo; $\mathrm{Fr}>85=$ Prominencia del síndrome.

Fuente: elaboración propia.

Respecto a los síndromes clínicos, el trastorno de ansiedad en comparación con los otros síndromes obtuvo la mayor puntuación con un porcentaje de

25.6 para presencia y 28.9 para prominencia; por otro lado, el $21 \%$ obtuvo una puntuación mayor a 75 en trastorno bipolar (véase Tabla 3).

Tabla 3.

Síndromes clínicos según conducta delictiva

\begin{tabular}{|c|c|c|c|c|c|c|c|}
\hline \multirow{3}{*}{$\begin{array}{l}\text { Síndromes clínicos } \\
\text { moderados }\end{array}$} & \multirow{3}{*}{ Puntuación } & \multicolumn{6}{|c|}{ Tipo de delito } \\
\hline & & \multicolumn{2}{|c|}{ Violento } & \multicolumn{2}{|c|}{ No Violento } & \multicolumn{2}{|c|}{ Total } \\
\hline & & $\mathrm{F}$ & $\%$ & $\mathrm{~F}$ & $\%$ & $\mathrm{~F}$ & $\%$ \\
\hline \multirow{3}{*}{$\begin{array}{l}\text { Trastorno de } \\
\text { ansiedad }\end{array}$} & $<75$ & 44 & 51.2 & 11 & 31.4 & 55 & 45.5 \\
\hline & $75-84$ & 23 & 26.7 & 8 & 22.9 & 31 & 25.6 \\
\hline & $>85$ & 19 & 22.1 & 16 & 45.7 & 35 & 28.9 \\
\hline \multirow{3}{*}{$\begin{array}{l}\text { Trastorno } \\
\text { somatomorfo }\end{array}$} & $<75$ & 86 & 100.0 & 34 & 97.1 & 120 & 99.2 \\
\hline & $75-84$ & 0 & 0.0 & 1 & 2.9 & 1 & 0.8 \\
\hline & $>85$ & 0 & 0.0 & 0 & 0.0 & 0 & 0.0 \\
\hline \multirow{3}{*}{ Trastorno bipolar } & $<75$ & 70 & 81.4 & 26 & 74.3 & 96 & 79.3 \\
\hline & $75-84$ & 14 & 16.3 & 4 & 11.4 & 18 & 14.9 \\
\hline & $>85$ & 2 & 2.3 & 5 & 14.3 & 7 & 5.8 \\
\hline \multirow{3}{*}{ Trastorno distímico } & $<75$ & 86 & 100.0 & 35 & 100.0 & 121 & 100.0 \\
\hline & $75-84$ & 0 & 0.0 & 0 & 0.0 & 0 & 0.0 \\
\hline & $>85$ & 0 & 0.0 & 0 & 0.0 & 0 & 0.0 \\
\hline \multirow{3}{*}{$\begin{array}{l}\text { Dependencia del } \\
\text { alcohol }\end{array}$} & $<75$ & 75 & 87.2 & 30 & 85.7 & 105 & 86.8 \\
\hline & $75-84$ & 9 & 10.5 & 5 & 14.3 & 14 & 11.6 \\
\hline & $>85$ & 2 & 2.3 & 0 & 0.0 & 2 & 1.7 \\
\hline \multirow{3}{*}{$\begin{array}{l}\text { Dependencia de } \\
\text { sustancias }\end{array}$} & $<75$ & 75 & 87.2 & 28 & 80.0 & 103 & 85.1 \\
\hline & $75-84$ & 7 & 8.1 & 6 & 17.1 & 13 & 10.7 \\
\hline & $>85$ & 4 & 4.7 & 1 & 2.9 & 5 & 4.1 \\
\hline \multirow{3}{*}{$\begin{array}{l}\text { Trastorno estrés } \\
\text { postraumático }\end{array}$} & $<75$ & 86 & 100.0 & 35 & 100.0 & 121 & 100.0 \\
\hline & $75-84$ & 0 & 0.0 & 0 & 0.0 & 0 & 0.0 \\
\hline & $>85$ & 0 & 0.0 & 0 & 0.0 & 0 & 0.0 \\
\hline
\end{tabular}

Nota. $\mathrm{Fr}<75=$ Ausencia; Fr 75 - 84= Presencia del síndrome; $\mathrm{Fr}>85$ = Prominencia del síndrome; $\%$ = Porcentaje

Fuente: elaboración propia. 
En cuanto a los síndromes clínicos graves se encuentra el trastorno delirante como el más presente y prominente, ubicándose en el $60 \%$ de los participantes (véase Tabla 4). En lo que respecta a depresión mayor, siete participantes puntuaron presencia del síndrome, evidenciando posible presencia de ideas suicidas.

Tabla 4.

Síndromes clínicos graves según conducta delictiva

\begin{tabular}{|c|c|c|c|c|c|c|c|}
\hline \multirow{3}{*}{$\begin{array}{l}\text { Síndromes } \\
\text { clínicos graves }\end{array}$} & \multirow{3}{*}{ Puntuación } & \multicolumn{6}{|c|}{ Tipo de delito } \\
\hline & & \multicolumn{2}{|c|}{ Violento } & \multicolumn{2}{|c|}{ No Violento } & \multicolumn{2}{|c|}{ Total } \\
\hline & & $F$ & & $F$ & & $F$ & \\
\hline \multirow{3}{*}{$\begin{array}{l}\text { Trastorno del } \\
\text { pensamiento }\end{array}$} & $<75$ & 73 & 84.9 & 30 & 85.7 & 103 & 85.1 \\
\hline & $75-84$ & 12 & 14.0 & 4 & 11.4 & 16 & 13.2 \\
\hline & $>85$ & 1 & 1.2 & 1 & 2.9 & 2 & 1.7 \\
\hline \multirow{3}{*}{ Depresión mayor } & $<75$ & 81 & 94.2 & 33 & 94.3 & 114 & 94.2 \\
\hline & $75-84$ & 5 & 5.8 & 2 & 5.7 & 7 & 5.8 \\
\hline & $>85$ & 0 & 0.0 & 0 & 0.0 & 0 & 0.0 \\
\hline \multirow{3}{*}{ Trastorno delirante } & $<75$ & 28 & 32.6 & 20 & 57.1 & 48 & 39.7 \\
\hline & $75-84$ & 50 & 58.1 & 8 & 22.9 & 58 & 47.9 \\
\hline & $>85$ & 8 & 9.3 & 7 & 20.0 & 15 & 12.4 \\
\hline
\end{tabular}

Nota. $\mathrm{Fr}<75=$ Ausencia; Fr $75-84=$ Presencia del síndrome; $\mathrm{Fr}>85=$ Prominencia del síndrome

Fuente: elaboración propia.

Como se evidencia en la Tabla 5, en patrones patológicos de personalidad, el $44 \%$ de la muestra obtuvo puntuaciones mayores a 75 en dos escalas; de igual forma, se identifica una prevalencia del 55\% para patrones patológicos de personalidad y del $36 \%$ para síndromes clínicos (véase tabla 5).

Tabla 5.

Total de escalas por cada participante según conducta delictiva

\begin{tabular}{lccccccc}
\hline & \multicolumn{7}{c}{ Tipo de delito } \\
\hline & Número de & \multicolumn{2}{c}{ Violento } & \multicolumn{2}{c}{ No violento } & \multicolumn{2}{c}{ Total } \\
\cline { 3 - 8 } & escalas & $>85$ & $75-85$ & $>85$ & $75-85$ & $>85$ & $75-85$ \\
\hline \multirow{2}{*}{$\begin{array}{l}\text { Patrones clínicos y } \\
\text { patologías graves de la }\end{array}$} & 1 & $44.2 \%$ & $41.9 \%$ & $48.6 \%$ & $42.8 \%$ & $45.4 \%$ & $42.1 \%$ \\
personalidad & 2 & $9.3 \%$ & $39.5 \%$ & $8.6 \%$ & $22.8 \%$ & $9.1 \%$ & $34.7 \%$ \\
& 3 & $0 \%$ & $1.2 \%$ & $0 \%$ & $0 \%$ & $0 \%$ & $0.8 \%$ \\
\hline & 1 & $18.6 \%$ & $46.5 \%$ & $28.6 \%$ & $28.6 \%$ & $21.5 \%$ & $41.3 \%$ \\
Síndromes clínicos & 2 & $8.1 \%$ & $17.4 \%$ & $20 \%$ & $31.4 \%$ & $11.6 \%$ & $21.5 \%$ \\
y síndromes clínicos & 3 & $2.3 \%$ & $7 \%$ & $5.7 \%$ & $5.7 \%$ & $3.3 \%$ & $6.6 \%$ \\
graves & 4 & $0 \%$ & $9.3 \%$ & $0 \%$ & $0 \%$ & $0 \%$ & $6.6 \%$ \\
\hline
\end{tabular}

Nota. $\mathrm{Fr}<75=$ Ausencia; Fr $75-84=$ Presencia de rasgos o síndrome; $\mathrm{Fr}>85=$ Presencia del trastorno o Prominencia del síndrome Fuente: elaboración propia. 
Finalmente, en cuanto al análisis correlacional, a partir del coeficiente ETA, el cual busca medir el nivel de asociación entre dos variables, una cuantitativa, definida como patrón patológico de personalidad y otra categórica, comprendida como tipo de delito; dicho coeficiente toma valores entre $0 \mathrm{y}$ 1 , indicando que cuando se aproxima a 0 , no hay asociación entre las variables; mientras que, sí es cercano a 1 se indica que hay gran relación entre las variables (Martin et al., 2008; Pedroza \& Dicovskyi, 2006; Sampieri et al., 2010).

En relación con los patrones clínicos de personalidad se encontraron correlaciones significativas en la mayoría de las escalas, las cuales se ubican entre .48 y .67. Con respecto a patologías graves de la personalidad; se identificaron correlaciones significativas en las escalas límite y paranoide, con un nivel de asociación de .57 (véase Tabla 6).

Tabla 6.

Correlación entre patrones clínicos de personalidad y tipo de delito

\begin{tabular}{lc}
\hline $\begin{array}{l}\text { Patrones clínicos y patologías } \\
\text { graves de la personalidad }\end{array}$ & $\begin{array}{c}\text { Nominal por } \\
\text { intervalo Eta }\end{array}$ \\
\hline Agresiva & .51 \\
Antisocial & .55 \\
Autodestructiva & .66 \\
Compulsiva & .67 \\
Dependiente & .57 \\
Depresiva & .62 \\
Esquizoide & .49 \\
Evitativa & .64 \\
Histriónica & .59 \\
Narcisista & .61 \\
Negativista & .51 \\
Esquizotípico & .48 \\
Límite & .57 \\
Paranoide & .57 \\
\hline
\end{tabular}

Fuente: elaboración propia.

\section{Discusión}

Respecto al análisis de la prevalencia de patrones de personalidad patológicos, se encontró con mayor frecuencia los patrones clínicos de personali- dad narcisista (21\% presencia rasgo y $38 \%$ presencia trastorno) y compulsivo (22\% presencia rasgo y $11 \%$ presencia trastorno), estos datos concuerdan con los hallazgos de Gondolf (1999), Vicens (2006) y Vicens-Pons (2009) en relación al patrón narcisista, ya que en estos también se encontró mayor prevalencia. De igual forma, Ortiz-Tallo y Sánchez (2002), Fernández-Montalvo y Echeburúa (2008) y Echauri, Fernández-Montalvo, Martínez y Azcárate (2011) encontraron que el patrón más prevalente fue el compulsivo, ya que se presentó entre el 58\% y el $61 \%$ de la población.

Por su parte López-Barrachina, Lafuente y GarcíaLatas (2007) encontraron puntuaciones elevadas en narcisista (60\%), compulsivo (64\%) y antisocial (47\%), estos datos difieren de los hallazgos de la presente investigación, debido a que en esta se obtuvo una puntuación del $6 \%$ en rasgos antisocial y por tanto, se ratifica lo mencionado por Millon \& Davis (1998), quienes ya habían afirmado que solo un reducido grupo de individuos con trastorno antisocial entran en conflicto con la ley, ya que estos logran mantenerse entre los límites de lo permitido socialmente. Al respecto, es de resaltar que los individuos narcisistas se caracterizan por su arrogancia, se consideran superiores y justifican sus comportamientos erróneos, evitando asumir responsabilidades, y por tanto, esto soporta lo afirmado por Roussos y Siever, (2012), en el sentido que estas personas no tienden a percibir la complejidad de su patología y sumado a esto, se sienten a gusto con su condición y no consideran que deban modificar su comportamiento (Vázquez, 2012). Por último, su conducta arrogante genera tendencia a burlarse de las normas e implicarse en acciones que pueden ocasionarle problemas con la justicia (Millon \& Davis, 1998), tal como se presenta en la población de estudio.

Del mismo modo, el trastorno de personalidad paranoide $(52.9 \%$ presencia rasgo y $10.1 \%$ presencia trastorno) se identificó como el más prevalente en relación a las patologías graves de la personalidad; esto concuerda con lo encontrado en las investigaciones de López-Barrachina et al. (2007), Fernández-Montalvo y Echeburúa (2008), VicensPons (2009) y Echauri et al. (2011), dado que identificaron puntuaciones elevadas en el trastornos paranoide, con un porcentaje de aparición entre el $30 \%$ y $41.1 \%$. 
En cuanto a las patologías graves de la personalidad, estas se agruparon independiente a los patrones clínicos de personalidad, ya que como se evidencia en los resultados, estos individuos representan las variantes disfuncionales más extremas de las patologías de la personalidad. Así, dada la elevada frecuencia e intensidad con la que se presentaron se evidenciaron rasgos como, comportamientos extraños, pensamiento peculiar, actitud desconfiada, explosión emocional, leve control interno, limitadas estrategias de afrontamiento, estado de ánimo frio o falto de alegría, estos ámbitos, tal como plantea Millon y Davis (1998), son rasgos que generan dificultades en el desarrollo de la capacidad para aprender de sus errores y presentan deterioro en las relaciones personales, en el rendimiento escolar y laboral. Por tal motivo, se implican en los mismos fracasos una y otra vez, sumado a esto, los individuos no logran captar el carácter engañoso de los estímulos internos, generando una presión interna que los lleva a desarrollar actos o pensamientos extraños y hostiles.

Por otro lado, diversos estudios se han delimitado a identificar la prevalencia general de los trastornos de personalidad, en esta investigación se identificó que esta fue del $54.4 \%$, cifra que se aproxima a la obtenida en los estudios de López-Barrachina et al.(2007) y Echauri et al. (2011), los cuales encontraron una prevalencia del $69.3 \%$ y del $79.3 \%$ respectivamente. Por otro lado, otros autores, determinaron prevalencias del 22\% (Calvo et al., 2008), 30\% (Arroyo \& Ortega, 2007), 40\% (Vicens, 2006), 80\% (Vicens-Pons, 2009), 86.6\% (FernándezMontalvo \& Echeburúa, 2008) y $90.08 \%$ (Sáez \& Fernández, 2000), las cuales difieren en mayor 0 menor medida con las nuestras.

Teniendo en cuenta dos factores importantes, en primer lugar, los altos niveles de prevalencia identificados en relación a los trastornos de personalidad, y en segundo lugar, el hecho que los individuos se encuentran en prisión, se generaron afectaciones a nivel cognitivo, comportamental y emocional, aumentando la probabilidad de exacerbar dicho cuadro patológico. Esto, dado que se aleja al individuo de las redes de apoyo, se les restringen los movimientos y los espacios y no tienen intimidad (Arroyo \& Ortega, 2009; Ruiz, 2007). Por tal moti- vo, es importante tener en cuenta que los centro de reclusión contribuyen a agravar el cuadro clínico, en especial en prisiones de máxima seguridad (Casares-López et al., 2010), además, tal como afirma Millon y Davis (1998), los patrones clínicos de personalidad son sistemas dinámicos, ya que los comportamientos, las conductas sociales, los procesos cognitivos y los mecanismos inconscientes van cambiando y trasformando constantemente, siendo modificados por el contexto donde se desarrolla.

Asimismo, un rasgo fundamental en los participantes que presentaron puntuaciones elevadas en patrones patológicos de personalidad, fue su incapacidad para adaptarse al medio, ya que como lo ratifica Millon (1969; citado en Millon et al., 2006), dichos individuos necesitan que su medio se adapte a ellos, si este no lo logra, al individuo se le dificulta el resolver situaciones que le ocasionan estrés subjetivo. Además, el manejo de las estrategias de afrontamiento se torna limitado, se afecta el establecimiento de relaciones interpersonales (Esbec \& Echebúrua, 2011), y se desarrollan conductas que promueven efectos desfavorables para la salud (Cardenal, Sánchez, \& Ortiz-Tallo, 2007).

Por otro lado, es importante tener en cuenta que dichos individuos no pueden ser diagnosticados de una forma definitiva, en primer lugar, se deben valorar las diferencias individuales para así poder diseñar intervenciones multidisciplinarias, que estén entrelazadas en el tiempo y tengan congruencia en su aplicación, ya que como lo plantean Millon \& Davis, (1998), una evaluación efectiva debe diferenciar los rasgos que se presentan con mayor frecuencia y configuran su comportamiento global. Esto debido a que los individuos, varían en el grado de aproximación a cada prototipo de personalidad patológica, buscando que en un proceso de terapia, se busque flexibilizar estos rasgos, permitiendo una mayor variedad de los estados o comportamientos adaptativos en distintas situaciones.

Por lo anteriormente señalado, se plantea una dificultad, ya que se considera que a la población reclusa se le debe estar brindando un adecuado servicio de salud mental, en el que se planteen tratamientos que promuevan el bienestar psicológico de los individuos, a razón de las alta cifras obtenidas en 
esta investigación, así como en diversos estudios, en relación a la prevalencia de patrones clínicos de personalidad. Sin embargo, la realidad es diferente, ya que como plantea Esbec \& Echeburúa (2010) y Marín-Basallote y Navarro-Repiso, (2012), la capacidad de atención y tratamiento de los servicios de salud mental en reclusos es limitada, generando que las patologías se tornen más críticas.

Otro aspecto importante, es el relacionado con los síndromes clínicos, puesto que como lo menciona Millon et al. (2009), las patologías de la personalidad se deben evaluar como un constructo multideterminado y multirreferencial, por ende, requieren una valoración de la naturaleza interactiva del sistema Multiaxial. Es decir, para dar explicación a los patrones clínicos de personalidad es necesario conocer la interrelación que existe con los síndromes clínicos, entendidos estos como estados claros o transitorios que intensifican los rasgos básicos de la personalidad. Teniendo en cuenta lo anterior, y, debido a la presencia en grado moderado y grave de los trastornos delirante $(47.9 \%)$ de ansiedad (28.9\%), del pensamiento (13.2), bipolar (5.8\%), depresión mayor $(5.8 \%)$, dependencia de sustancias $(4.1 \%)$, $\mathrm{y}$, en grado prominente, de los trastornos de ansiedad (25.6\%), bipolar (14.9\%), delirante $(12.4 \%)$, dependencia de alcohol (11.6\%) y dependencia de sustancias (4.1\%). Estos hallazgos concuerdan con lo planteado por Vicens (2006), quien identificó en el $7 \%$ de los participantes, presencia del trastorno bipolar. Asimismo, Black, Gunter, Loveless, Allen, y Sieleni (2010), plantean índices elevados en ansiedad y consumo de sustancias (Black et al., 2010). Por su parte, estudios como el de Ortiz-Tallo, Fierro, Blanca, Cardenal, y Sánchez (2006) y Casares-López et al., (2010) encontraron el trastorno del pensamiento y trastorno delirante como síndromes prominentes y en baja medida el consumo de sustancias.

Un factor asociado a los altos índices de prevalencia en patrones patológicos de personalidad, puede ser el limitado o deficiente manejo de las estrategias de afrontamiento, ya que, como indican Millon \& Davis (1998), esta situación incrementa exponencialmente la probabilidad de presentar síndromes clínicos, tales como trastorno de ansiedad o depresión. Esto, debido a que los individuos tienden a desarrollar círculos viciosos que intensifican las dificultades, debilitando así los sistemas que predisponen al desarrollo de dichos síntomas. Lo último se explica en los datos obtenidos en la presente investigación, dadas las altas puntuaciones en síndromes clínicos, especialmente en las escalas de trastorno delirante, trastorno de ansiedad y trastorno del pensamiento; de igual forma, los altos índices de correlación que se encontraron entre patrones clínicos y patología grave de personalidad con síndromes clínicos moderados y graves.

Finalmente, en cuanto a la relación entre las variables de estudio, se da soporte a la hipótesis de investigación, puesto que a través del coeficiente de correlación ETA se encontraron correlaciones entre .48 y .67 , con un promedio de .57 , siendo las más significativas, las que se ubican en los patrones patológicos de personalidad compulsivo (.67), autodestructivo (.66), y evitativo (.64). Esto a su vez, corrobora lo plateado por Esbec \& Echeburúa (2010), quienes le brindan a la variable: patrón clínico de personalidad un carácter predictivo, mencionando que, a partir de esta, se pueden identificar las conductas criminales que un individuo puede llegar a desarrollar. Sin embargo, es importante resaltar que hasta el momento no se identifican estudios similares y por tanto se convierte en un tema de interés para futuras investigaciones.

\section{Referencias}

Arroyo, J. \& Ortega, E. (2007). Un programa de mejora de la calidad asistencial de los problemas de salud mental en prisión. Evaluación de resultados después de 6 años (2000-2005). Revista Española de Sanidad Penitenciaria, 9(1), 11-20.

Arroyo, J. \& Ortega, E. (2009). Los trastornos de personalidad en reclusos como factor de distorsión del clima social de la prisión. Revista Española de Sanidad Penitenciaria, 11(1), 11-15.

Black, D., Gunter, T., Loveless, P., Allen, J., \& Sieleni, B. (2010). Antisocial pesonality disorder in incarcerated offenders: Psychiatric comorbidity and quality of life. Annals of Clinical Psychiatry, 22(2), 113-120.

Calvo, P., Soler, C., Día, J., \& Ventura, T. (2008). Prevalencia de trastornos psiquiátricos en 
pacientes ingresados por el Servicio de Psiquiatría en el Módulo Penitenciario del H.U.M.S. Revista Española de Sanidad Penitenciaria, 10.lib, 69-72.

Cardenal, V., Sánchez, M., \& Ortiz-Tallo, M. (2007). Los trastornos de personalidad según el modelo de Millón: una propuesta integradora. Clínica y Salud, 18(3), 305-324.

Casares-López, M., González-Menéndez, A., Torres-Lobo, M., Secades-Villa, R., FernándezHermida, J., \& Álvarez, M. (2010). Comparación del perfil psicopatológico y adictivo de dos muestras de adictos en tratamiento: En prisión y en comunidad terapéutica. International Journal of Clinical and Health Psychology, 10(2), 225-243.

Corte Constitucional Colombiana. (2000). Código Penal Colombiano. Bogotá: Legis.

De Barbenza, C. (2003). El abordaje integrativo de la personalidad en la teoría de Theodore Millon. Centro Interamericano de Investigaciónes Psicológicas y Ciencias Afines, 20(1), 61-74.

Echauri, J., Fernández-Montalvo, J., Martínez, M., \& Azcárate, J. (2011). Trastornos de Personalidad en Hombres Maltratadores a la Pareja: Perfil Diferencial entre Agresores en Prisión y Agresores con Suspensión de Condena. Anuario de Psicología Jurídica, 21, 97-105.

Echeverry, J., Escobar, F., Martínez, J., Garzón, A., \& Gómez, D. (2002). Trastorno de personalidad antisocial en condenados por homicidio en Pereira, Colombia. Investigación En Salud, 4(2), 1-10

Esbec, E. \& Echeburúa, E. (2010). Violencia y trastornos de la personalidad: implicaciones clínicas y forenses. Actas Españolas de Psiquiatría, 38(5), 249- 261.

Esbec, E. \& Echebúrua, E. (2011). La reformulación de los trastornos de la personalidad en el DSM5. Actas Españolas de Psiquiatría, 39(1), 7-17.

Fernández-Montalvo, J. \& Echeburúa, E. (2008). Trastornos de personalidad y psicopatía en hombres condenados por violencia grave contra la pareja. Psicothema, 20(2), 193-198.

Gondolf, W. (1999). MCMI-III Results for Batterer Program Participants in Four Cities: Less "Pathological" Than Expected. Journal of Family Violence, 14(1), 1-17.

González-Guerrero, L. (2007). Características descriptivas de los delitos cometidos por sujetos con trastornos de la personalidad: motivaciones subyacentes, "modus operandi" y relaciones víctima-victimario. Psicopatología Clínica Legal y Forense, 7(1), 19-39.

López-Barrachina, R., Lafuente, O., \& García-Latas, J. (2007). Del mito de Narciso a los trastornos de la personalidad en las cárceles aragonesas: Una aproximación al perfil de estos desórdenes en las personas privadas de libertad. Revista Española de Sanidad Penitenciaria, 9, 53-63.

Marco, R., Benítez, M., \& Morera, A. (2006). Conducta suicida en trastornos de personalidad. Revista Española de Sanidad Penitenciaria, 8(1), 108-111.

Marín-Basallote, N. \& Navarro-Repiso, C. (2012). Estudio de la prevalencia de trastorno mental grave (TMG) en los centros penitenciarios de Puerto I , II y III del Puerto de Santa María (Cádiz ): nuevas estrategias en la asistencia psiquiátrica en las prisiones. Revista Española de Sanidad Penitenciaria, 14 (3), 80-85.

Martin, Q., Cabero, M., \& Santana, Y. (2008). Tratamiento estadístico de datos con SPSS. (C. Fuente, Ed.) (Primera Ed). Madrid: Thomson.

Millon, T. \& Davis, R. (1998). Trastornos de la personalidad. Mas allá del DSM-IV (2da ed.). España: Masson.

Millon, T., Davis, R., \& Millon, C. (2009). Inventario Clinico Multiaxial de Millon-III (Segunda). Madrid: TEA Ediciones.

Ortiz-Tallo, M., Fierro, A., Blanca, M., Cardenal, V., \& Sánchez, L. (2006). Factores de personalidad y delitos violentos. Psicothema, 18(3), 459-464. 
Ortiz-Tallo, M. \& Sánchez, L. (2002). Perfil psicológico de delincuentes sexuales. Un estudio clínico con el MCMI-I. Revista de Psiquiatría de La Facultad de Medicina, 29(3), 144-152.

Pedroza, D. \& Dicovskyi, L. (2006). Sistema de análisis estadístico con SPSS. (E. Rodríguez, Ed.) (Primera Ed). Managua: Instituto Nicaraguense de Tecnología Agropecuaria.

Rodríguez, A., López, J., \& Pueyo, A. (2002). Personalidad y comportamiento penitenciario. Psicothema, 14 (1), 90-100.

Rodríguez-Arrebola, E. (2005). Abordaje psiquiátrico de los trastornos de personalidad en el medio penitenciario. Revista Española de Sanidad Penitenciaria, 7 (3), 134-141.

Roussos, P. \& Siever, L. (2012). Neurobiological Contributions. En T. Widigert (Ed.), The Oxford Handbook of Personality Disorders (Oxford Uni, pp. 299-324). New York: Oxford.

Ruiz, J. (2007). Sintomas psicológicos, clima emocional, cultura y factores psicosociales en el medio penitenciario. Revista Latinoamericana de Psicología, 39(3), 547-561.

Sáez, C. \& Fernández, P. (2000). Tasas de Prevalencia y Co-Ocurrencia de Trastornos DSMIv y Dimensiones de Personalidad en Reclusos Sometidos a Proceso Criminal. Universidad Santo Tomás. http://www.psicologia-online. com/colaboradores/paola/Bibliografia.htm
Sáez, C. \& Silvia, P. (2000). Tasas de Prevalencia y Coocurrencia de Trastornos DSM IV - Dimensiones de Personalidad en Reclusos Sometidos a Proceso Criminal. Universidad Santo Tomás. http://www.psicologia-online.com/colaboradores/paola/Metodologia.htm

Sampieri, R., Collado, C., \& Baptista, M. (2010). Metodología de la investigación. (J. Chacón, Ed.) (5ta ed.). Ciudad de México: The McGraw.Hill.

Soria, M. (2006). La Psicologia criminal: desarrollo conceptual y ámbitos de aplicación. En Psicología Criminal (3. ${ }^{a}$ ed., pp. 24-119). España: Pearson.

Vázquez, S. (2012). Trastornos de la Personalidad y Conducta Delictiva. Informe Instituto de Ciencias Forenses y de la seguridad. Madrid: Universidad Autónoma.

Vicens, E. (2006). Violencia y enfermedad mental. Revista Española de Sanidad Penitenciaria, 8 (3) 95-99.

Vicens-Pons, E. (2009). Aproximación a la Metodología para el estudio de los Trastornos mentales en población penitenciaria: El estudio PreCa. Revista Española de Sanidad Penitenciaria, 1(1), 17-25.

Vidal, L., Acosta, M., \& Caridad, M. (2004). Trastornos de personalidad y su relación con la violencia. Revista de Psiquiatria, 8(3), 2-8. 